\title{
Low expression of novel IncRNA RP11-462C24.1 suggests a biomarker of poor prognosis in colorectal cancer
}

\author{
Debing Shi $\cdot$ Hongtu Zheng $\cdot$ Changhua Zhuo $\cdot$ \\ Junjie Peng • Dawei $\mathrm{Li} \cdot \mathrm{Ye} \mathrm{Xu} \cdot$ Xinxiang $\mathrm{Li} \cdot$ \\ Guoxiang Cai $\cdot$ Sanjun Cai
}

Received: 9 April 2014/ Accepted: 9 May 2014/Published online: 8 June 2014

(C) The Author(s) 2014. This article is published with open access at Springerlink.com

\begin{abstract}
Long noncoding RNAs (lncRNAs) have recently emerged as a major class of regulatory molecules, which were involved in a broad range of biological processes and complex diseases. Research on lncRNAs may shed light on tumorigenesis and progression of colorectal cancer (CRC). The purpose of the present study was to identify lncRNAs correlated with CRC and then investigate their potential functions. We selected 92 patients for this prospective study and then collected the tumor samples and clinical records. First, the global lncRNA expression profiles in tumor and adjacent normal tissues of patients with non-metastatic CRC and patients with metastatic CRC were measured by microarray assay. Then, a noteworthy lncRNAs RP11-462C24.1 whose function was previously unknown was explored in detail on the aspect of the association of its expression level and clinicopathological
\end{abstract}

Debing Shi and Hongtu Zheng contribute equally to this study.

Electronic supplementary material The online version of this article (doi:10.1007/s12032-014-0031-7) contains supplementary material, which is available to authorized users.

D. Shi · H. Zheng · C. Zhuo - J. Peng · D. Li · Y. Xu · X. Li ·

G. Cai $(\bowtie) \cdot$ S. Cai $(\bowtie)$

Department of Colorectal Surgery, Fudan University Shanghai

Cancer Center, Shanghai 200032, China

e-mail: gxcai@fudan.edu.cn

S. Cai

e-mail: caisanjun@gmail.com

D. Shi

e-mail: shidebing7819@163.com

D. Shi - H. Zheng - C. Zhuo - J. Peng - D. Li · Y. Xu - X. Li

G. Cai - S. Cai

Department of Oncology, Shanghai Medical College,

Fudan University, Shanghai 200032, China features of CRC and patients' survival. We found that RP11-462C24.1 expression level was lower in cancer tissues compared with adjacent normal samples $(P<0.001)$. Furthermore, its expression level was lower in CRC patients with metastasis than those without metastasis $(P=0.049)$. That is, RP11-462C24.1 expression level decreased as the malignant degree of CRC increased. In addition, low expression of RP11-462C24.1 significantly correlated with more distant metastasis $(P=0.011)$. The areas under ROC curves were 0.78 and 0.65 for RP11462C24.1, distinguishing CRC from normal tissue and distinguishing CRC without metastasis from CRC with metastasis, respectively. Multivariate analysis identified that RP11-462C24.1 was an independent predictor for patients prognosis $(P=0.005)$. Furthermore, KaplanMeier analysis showed that patients with low expression of RP11-462C24.1 had a poor disease-free survival $(P<0.001)$. This is the first study that correlates RP11$462 \mathrm{C} 24.1$ expression profile with malignancy grade in human CRC. Our results showed that RP11-462C24.1 could be a potential novel prognostic marker for CRC, and thus, provided a new strategy for CRC diagnosis. Meanwhile, our findings indicated the potential roles of RP11$462 \mathrm{C} 24.1$ in tumorigenesis and progression of CRC, which gave a clue for future studies.

Keywords Colorectal cancer - Long noncoding RNAs · Microarray $\cdot$ RP11-462C24.1 · Survival

\section{Introduction}

Colorectal cancer (CRC), which ranks the third in the cancer morbidity and the second in the cancer mortality, is the most prevalent malignant cancer in the world with 
annual new cases exceeding 1,000,000 [1]. Although great progresses have been made in therapy of CRC, 30-40\% patients still died of relapse and metastasis, among which liver metastasis is the leading cause of death [2-4]. Therefore, it is crucial to accurately diagnose liver metastasis in early stage and then following a preventive treatment. Currently, the diagnosis for relapse and metastasis and therapy strategies are mostly based on tumor node metastasis (TNM) stages [5, 6]. However, this method always leads to a misdiagnosis, as it excludes the underlying molecular mechanisms, which were responsible for cancer progression [7]. Thus far, more and more studies are looking for biomarkers to employ them in the early diagnosis of liver metastasis and accurate prognosis for patients with CRC.

Long noncoding RNA (lncRNA) is a type of RNA molecule with length of more than $200 \mathrm{bp}$ and lacks an open reading frame of significant length and the capability of coding protein [8-11]. When it comes to the structural similarity to mRNA, both kinds of RNAs are marked by $5^{\prime}$ cap and $3^{\prime}$ poly A. However, it has a diverse subcellular location and plays important roles in many aspects of cell activity. As a new type of regulatory RNA molecule, lncRNAs exert the role of regulating gene expression in various layers of epigenetics, transcription, post-transcription and translation during the development of cancer [12, 13]. Based on their genomic proximity to protein-coding genes, lcnRNAs are mainly classified into five types: sense, antisense, bidirectional, intronic, and intergenic [10, 14].

Cancer is a complex disease involving multiple changes in gene expression. To date, researchers are mainly interested in protein-coding genes, which indeed have been shown to affect cancer progression. However, the underlying mechanisms of cancer are far from complete elucidation. With the discovery of IncRNA, it provided a new insight into revealing uncharacterized aspects of cancer biology. Recently, several studies have demonstrated that lncRNAs are not only involved in the origin and development of human diverse cancers, but also as potential biomarkers for the prognosis for CRC patients. HOX Antisense Intergenic RNA (HOTAIR) was reported to be a negative prognostic factor for breast, colon, liver, and pancreatic cancer patient survival and involved in chromatin remodeling by interaction with polycomb repressive complex 2 (PRC2) [15-19]. Metastasis-associated lung adenocarcinoma transcript 1(MALAT1), also known as NEAT2 (nuclear-enriched abundantly transcript 2), was found to be overexpressed not only in early-stage metastasizing non-small cell lung cancer (NSCLC) but also in breast, pancreas, colon, prostate, and liver cancers [20, 21]. It was involved in regulated alternative splicing and transcriptional activity [22, 23]. More important, patients with high expression level of MALAT1 have poor prognosis
[21]. Colorectal neoplasia differentially expressed (CRNDE), an up-regulated lncRNA in colorectal cancer, glioma, and leukemia, was reported with complex alternative splicing and multifunctional in tumorigenesis [2427]. However, the relationship between IncRNA expression level and progression of CRC is still elusive.

In the present study, we aim to find new IncRNAs that correlated with CRC and test whether the expression of those lncRNA could be a potential biomarker for CRC. Specifically, we first want to identify all aberrantly expressed lncRNAs in CRC, by applying microarray assay. Second, we further investigated the expression pattern and genomic location of these dysregulated lncRNAs. Then, six aberrantly lncRNAs (ENST00000428029, ENST00000423943, RP11-462C24.1, AK097793, ENST00000393516 and uc002wvk.2) were selected and further confirmed their expression pattern by RT-PCR assay. Finally, we focused on one lncRNA, RP11-462C24.1, which could be a biomarker for CRC, for the analysis of association between its expression and clinical characteristics and association between its expression and patients' survival.

\section{Materials and methods}

CRC samples and clinical data collection

A total of 92 patients were selected in this prospective study. Six of them were involved in a microarray assay to figure out lncRNAs aberrantly expressed in CRC. Specimens underwent resection of the primary CRC at Fudan University Shanghai Cancer Center. All the diagnoses of CRC were histopathologically confirmed. No patient has received preoperative treatment. Resected tissue samples were immediately frozen in liquid nitrogen and stored at $-80{ }^{\circ} \mathrm{C}$ until RNA extraction. The data collected on all subjects, including age, gender, disease-specific survival (DSS) and CRC features, such as tumor size, location, histological stage, depth of invasion, and the status of liver metastasis; detailed information were given in Supplementary Material 1. Clinical stage of CRC was evaluated based on the TNM classification system [28, 29]. Patients follow-up were performed every 2-3 months during the first year after surgery and 3-6 months thereafter until November 30, 2012. All the 92 patients had completed follow-up, and 21 patients died of CRC. The DSS was defined as the length of time between the surgery and death specifically from the cancer.

Ethics statement

This study was conducted according to the principles expressed in the Declaration of Helsinki. Tissue specimen 
collections were made with full informed consent of the patients and following institutional ethical guidelines that were reviewed and approved by the ethics committee at the hospital clinical ethics committee (ID 050432-4-1212B), Fudan University, Shanghai, China.

\section{Microarray assay}

Six patients were involved in microarray assay. Of which, three patients were diagnosed as CRC without metastasis, whereas other three patients were diagnosed as CRC with liver metastasis. Specifically, cancer tissues from three patients with non-metastatic CRC were pooled and hybridized to one chip. Matched adjacent normal tissues from the above identical three patients were pooled and hybridized to second chip. Cancer tissues from the remaining three patients with non-metastatic CRC were pooled and hybridized to third chip. It thus allowed us to roughly detect the dysregulated lncRNAs between CRC tumor tissues and adjacent normal tissues and between CRC without metastasis and CRC with metastasis. Details about pooling information are described in Supplementary Material 2. Total RNA was extracted using TRIzol (Invitrogen, Carlsbad, CA, USA), following the manufacturers' protocol. RNA preparation was cleaned up by RNeasy ${ }^{\circledR}$ MinElute ${ }^{\mathrm{TM}}$ Cleanup Kit (Qiagen, Valencia, CA, USA). RNA integrity was assessed by standard denaturing agarose gel electrophoresis. One micro liter of total RNA from each sample was amplified and transcribed into fluorescent cRNA along the entire length of the transcripts without bias utilizing a random priming method, and cRNA was labeled and hybridized to the Human lncRNA Array v2.0 $(8 \times 60 \mathrm{~K}$, Arraystar). In total, 33,045 lncRNAs and 30,215 coding transcripts, which were collected from the most authoritative databases such as Reference Sequence, University of California, Santa Cruz (UCSC), Knowngenes, Ensembl and many related literatures can be detected by the microarray. After washing slides, the arrays were scanned by the Agilent Scanner G2505B and the acquired array images were analyzed by Agilent Feature Extraction software (version 10.7.3.1). Quantile normalization and subsequent data processing were performed using the GeneSpring GX v11.5.1 software package (Agilent Technologies).

\section{Quantitative real-time PCR}

To further confirm the generality of expression pattern of six lncRNAs in human population, specimens from 86 patients, who comprised of 36 patients without metastatic $\mathrm{CRC}$ and 50 patients with metastatic CRC, were used to perform qRT-PCR assay. Total RNA was extracted from frozen specimens using TRIzol reagent (Invitrogen Life
Technologies). The $20 \mu \mathrm{L}$ RT reactions were performed using a PrimeScript ${ }^{\circledR}$ RT reagent Kit (Takara, Dalian, China) and incubated for $30 \mathrm{~min}$ at $37{ }^{\circ} \mathrm{C}, 5 \mathrm{~s}$ at $85^{\circ} \mathrm{C}$ and then maintained at $4{ }^{\circ} \mathrm{C}$. For real-time PCR, $1 \mu \mathrm{L}$ diluted RT products were mixed with $10 \mu \mathrm{L}$ of $2 \times \mathrm{SYBR}^{\circledR}$ Premix Ex $\operatorname{Taq}^{\mathrm{TM}}$ (Takara, Dalian, China), $0.6 \mu \mathrm{L}$ forward and reverse primers $(10 \mu \mathrm{M})$, and $8.4 \mu \mathrm{L}$ nuclease-free water in a final volume of $20 \mu \mathrm{L}$, according to manufacturer's instructions. The primers used in this study are included in Supplementary Material 3. All reactions were run on the Eppendorf Mastercycler EP Gradient S (Eppendorf, Germany), using the following protocol: one cycle at $95{ }^{\circ} \mathrm{C}$ for $3 \mathrm{~min} ; 40$ cycles of $95{ }^{\circ} \mathrm{C}$ for $15 \mathrm{~s}$, and $60{ }^{\circ} \mathrm{C}$ for $60 \mathrm{~s}$. The specificity of the PCR amplification was validated by the presence of a single peak in the melting curve analyses. Each RT-qPCR experiment was repeated three times. Relative expression of genes was calculated using the comparative cycle threshold (CT) $\left(2^{-\Delta \Delta C T}\right)$ method with glyceraldehyde-3-phosphate dehydrogenase (GAPDH) as the endogenous control.

Statistical analysis

All statistical analyses were performed using $\mathrm{R}$ software (http://www.r-project.org/). Correlation between RP11$462 \mathrm{C} 24.1$ expression and clinical factors was tested using $\chi^{2}$ tests. Univariate analysis was used to explore clinicopathological factors (i.e., age, gender, tumor size, tumor stage, invasion length) related with disease-specific survival. Then, significant clinicopathological factors, which were measured in univariate analysis, were further selected for multivariate analysis to reduce related factors. Cox proportional hazards analysis was applied to calculate the hazard ratio (HR) and the $95 \%$ confidence interval (CI). In addition, the Kaplan-Meier survival analysis was applied to test the correlations between expression level and patients' survival. The log-rank test was performed to test the statistical differences between survival curve of patients with lower RP11-462C24.1 expression level and that of patients with higher RP11-462C24.1 expression level. The mean value was used as classification threshold when necessary in $\chi^{2}$ tests and univariate/multivariate analysis. A two-tailed $P$ value of 0.05 or less was considered statistically significant.

\section{Results}

Overview of lncRNAs expression profile in CRC

To identify lncRNAs specifically dysregulated in CRC, we firstly compared expression profile between CRC tissues 
and adjacent normal tissues. A total of 5,963 lncRNAs demonstrated differential expressions (fold change $\geq 2$ ) between tumor tissues and adjacent normal tissues from non-metastatic CRC patients. In total, 3,029 lncRNAs were over-expressed, whereas 2,934 lncRNAs were down-regulated in CRC tumor tissues. The AC092165.4 was the most over-expressed IncRNA and followed by AP000525.8, PNAS-108, lincRNA-NANOS3-2, BC016035, AK057037 and AL137280. However, the AK024585 was the most down-regulated lncRNAs and followed by RP11-561O23.7, BC032913, AC007225.1, RP11-183K14.2, AC009133.1, and DQ597482. The detailed information is in Table 1.

We further compared expression profile between CRC tissues with metastasis and CRC tissues without metastasis. In total, there were 30,605 IncRNAs, which shown different expression pattern, consisting of 27,549 up-regulated and 3,056 down-regulated lncRNAs. The XIST was the most up-regulated lncRNA and followed by TRIM78P, RP11-753E22.3, DQ597482, CH17-12M21.1, RP11-598F17.1, CFLP4, CFLP3, AL358913.1, VDAC1P4, CTD-2244C20.1, KRT8P25, and KRT8P7, whereas the RP11-397E7.1 was the most downregulated lncRNA and followed by RP1-125N5.2, AK098474, BX322613.3, AX747988, RP11-543D5.3, lincRNA-KLF4-4, AC008625.2, lincRNA-BBOX1-2, AL137280, AK091806, BC016035, RP11-506D12.1, lincRNA-NANOS3-2, RP11128M1.1, CTGLF11P, CR620692, and RP11-630I5.1. The detailed information for top 16 lncRNA, which showed significantly difference in expression, was provided in Table 1. The outputs from microarray trails have been submitted to the GEO database with accession No. GSE52413.

RP11-462C24.1 was down-regulated in CRC

Of all the differentially expressed lncRNAs indicated by lncRNA expression profiling, six lncRNAs (ENST000 00428029, ENST00000423943, RP11-462C24.1, AK097793, ENST00000393516, and uc002wvk.2) were selected because of their expression levels and genomic locations. Unsupervised hierarchical clustering was constructed using MeV (Multi Experiment Viewer) v4.9 [30] to analyze the expression profile of three groups of samples (Fig. 1). Their expression levels were further confirmed in additional cohort of 86 samples, which comprised of 36 patients without metastatic CRC and 50 patients with metastatic CRC, using real-time PCR assay. Except ENST00000 423943, other five lncRNAs showed the consistent pattern. We then focused on RP11-462C24.1, because it was downregulated in $\mathrm{CRC}$ tissues compared with $\mathrm{CRC}$ adjacent normal tissues $(P<0.001)$ and even lower expressed in CRC tissues with metastasis than CRC tissues without metastasis ( $P=0.049$; Fig. 2), implying RP11-462C24.1 may play a role in tumor suppression.
Table 1 Parts of differentially expressed lncRNAs between CRC tissues and adjacent normal tissues and between metastatic and nonmetastatic CRC samples

\begin{tabular}{|c|c|c|}
\hline GeneSymbol & Regulation & Fold change \\
\hline \multicolumn{3}{|c|}{ Non-metastatic CRC versus adjacent sample } \\
\hline AC092165.4 & Up & 131 \\
\hline AP000525.8 & Up & 100 \\
\hline PNAS-108 & Up & 50 \\
\hline lincRNA-NANOS3-2 & Up & 30 \\
\hline BC016035 & Up & 29 \\
\hline AK057037 & Up & 28 \\
\hline AL137280 & Up & 24 \\
\hline AL137281 & Up & 22 \\
\hline AK024585 & Down & 46 \\
\hline RP11-561O23.7 & Down & 43 \\
\hline ВC032913 & Down & 32 \\
\hline AC007225.1 & Down & 30 \\
\hline RP11-183K14.2 & Down & 26 \\
\hline AC009133.1 & Down & 25 \\
\hline MTB & Down & 25 \\
\hline DQ597482 & Down & 21 \\
\hline \multicolumn{3}{|c|}{ Metastatic versus non-metastatic CRC sample } \\
\hline XIST & $\mathrm{Up}$ & 445 \\
\hline TRIM78P & Up & 75 \\
\hline RP11-753E22.3 & $\mathrm{Up}$ & 71 \\
\hline DQ597482 & $\mathrm{Up}$ & 68 \\
\hline XIST & Up & 58 \\
\hline CH17-12M21.1 & $\mathrm{Up}$ & 50 \\
\hline RP11-598F17.1 & Up & 49 \\
\hline CFLP4 & $\mathrm{Up}$ & 48 \\
\hline RP11-397E7.1 & Down & 92 \\
\hline RP1-125N5.2 & Down & 35 \\
\hline AK098474 & Down & 32 \\
\hline BX322613.3 & Down & 31 \\
\hline AX747988 & Down & 29 \\
\hline RP11-543D5.3 & Down & 28 \\
\hline lincRNA-KLF4-4 & Down & 28 \\
\hline AC008625.2 & Down & 27 \\
\hline
\end{tabular}

RP11-462C24.1 expression showed significant difference between CRC tumor tissues and adjacent normal tissue

To explore whether RP11-462C24.1 can serve as a biomarker to distinguish CRC from normal tissue, we constructed a receiver operating characteristic (ROC) curve by grouping all tumor samples, including samples with metastasis and without metastasis into one class and then grouping all normal samples into another class. RP11-462C24.1 expression level obtained from RT-PCR 


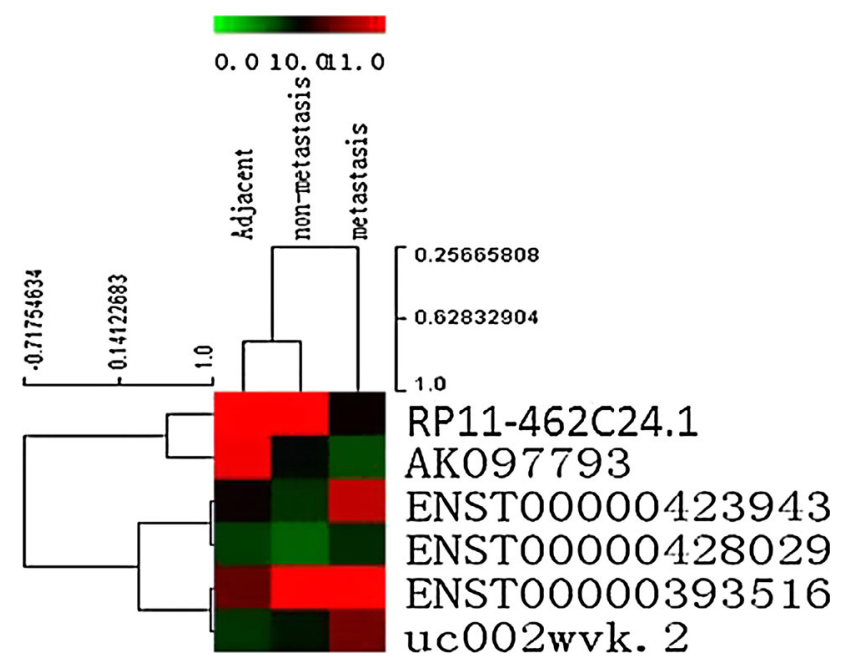

Fig. 1 Unsupervised hierarchical clustering of samples with 6 lncRNAs

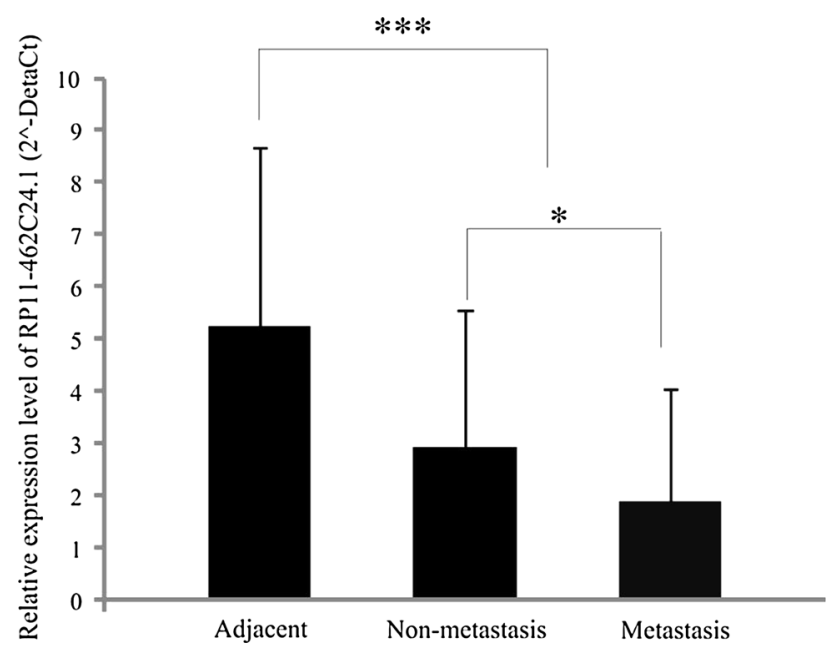

Fig. 2 Relative expression of RP11-462C24.1 in metastatic, nonmetastatic, and adjacent samples. RP11-462C24.1 expression was validated in 86 pairs of colorectal cancer and adjacent normal tissues by Q-RT-PCR, which was composed of 36 pairs of non-metastasis colorectal cancer samples and 50 pairs of metastasis samples. The height of the columns in the chart represent the mean expression value of $2^{-\Delta C \mathrm{t}}\left(\Delta C_{\mathrm{t}}=C_{\mathrm{t}}(\mathrm{RP} 11-46 \mathrm{C} 24.1)-C_{\mathrm{t}}(\mathrm{GAPDH})\right)$; The bars represent standard errors. $* P<0.05, * * * P<0.001$

data of a cohort of 86 patients mentioned above. The areas under ROC curve (AUC) of this ROC are 0.78 (Fig. 3a). Then, we constructed a ROC using all tumor samples alone by taking CRC samples with metastasis as one group and CRC samples without metastasis as another group. AUC of this ROC are 0.65 (Fig. 3b). Both ROC curves implied RP11-462C24.1 has a potential diagnostic value in CRC.
Correlation between RP11-462C24.1 expression and clinical characteristics

We assessed the correlations between RP11-462C24.1 expression and clinical characteristics by labeling the expression levels of RP11-462C24.1 in CRC tissue as low or high in relation to the mean value. RP11-462C24.1 expression level obtained from RT-PCR data of a cohort of 86 patients mentioned above. Of the 86 patients with CRC, 23 patients were classified into high RP11-462C24.1 group, and the remaining 63 patients were classified into low RP11462C24.1 group. RP11-462C24.1 expression level was significantly correlated with distant metastasis $(P=0.011$; Table 2). However, RP11-462C24.1 expression level was not significantly associated with other clinical factors, such as age $(P=0.132)$, gender $(P=0.300)$, tumor size $(P=0.180)$, depth of invasion $(P=0.372)$ or tumor stage $(P=0.070$; Table 2$)$.

Association between RP11-462C24.1 expression and patients' survival

To explored factors responsible for patients' survival, univariate and multivariate analysis were carried out. RP11-462C24.1 expression level obtained from RT-PCR data of a cohort of 86 patients mentioned above. Univariate analysis of disease-specific survival (DSS) revealed that metastasis $(P=0.0005)$, tumor stage $(P=0.015)$, and RP11-462C24.1 expression $(P=0.002)$ were prognostic indicators (Table 3). Multivariate analysis showed that the expression of RP11-462C24.1 $(P=0.005)$ and metastasis $(P=0.020)$ were independent prognostic indicators for survival of patients with CRC (Table 4). Furthermore, Kaplan-Meier analysis demonstrated that patients with low expression of RP11-462C24.1 had a significantly poor prognosis than those with high RP11-462C24.1 expression $(P<0.001$; Fig. 4).

\section{Discussion}

Colorectal cancer is a common malignancy tumor, which on the top in the new cases in China [24]. Despite curative surgical resection of the primary tumor, $40-50 \%$ of the patients ultimately die of metastasis [25]. However, the existence of multiple known carcinogens and varying genetic backgrounds makes it difficult to determine, which factors are most important in the development of CRC. Although many molecular markers, including carcinoembryonic antigen (CEA), have been exploited for CRC diagnosis, they lack sensitivity and specificity of evaluating the prognosis of CRC patient [31]. Thus, it is crucial to identify new biomarker responsible for predicting 
RP11-462C24.1 AUC $=77.83 \%$

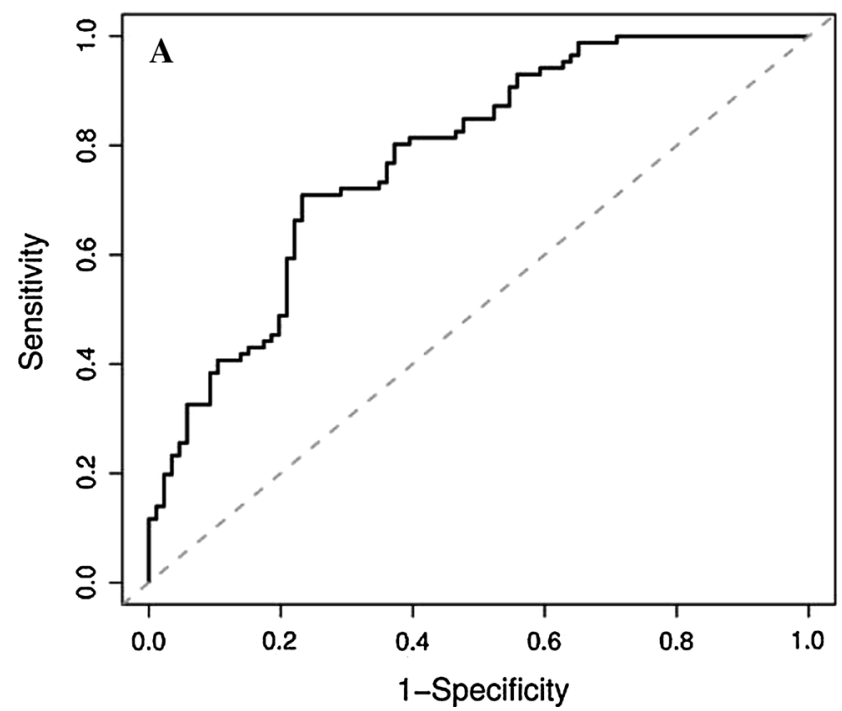

RP11-462C24.1 AUC $=64.5 \%$

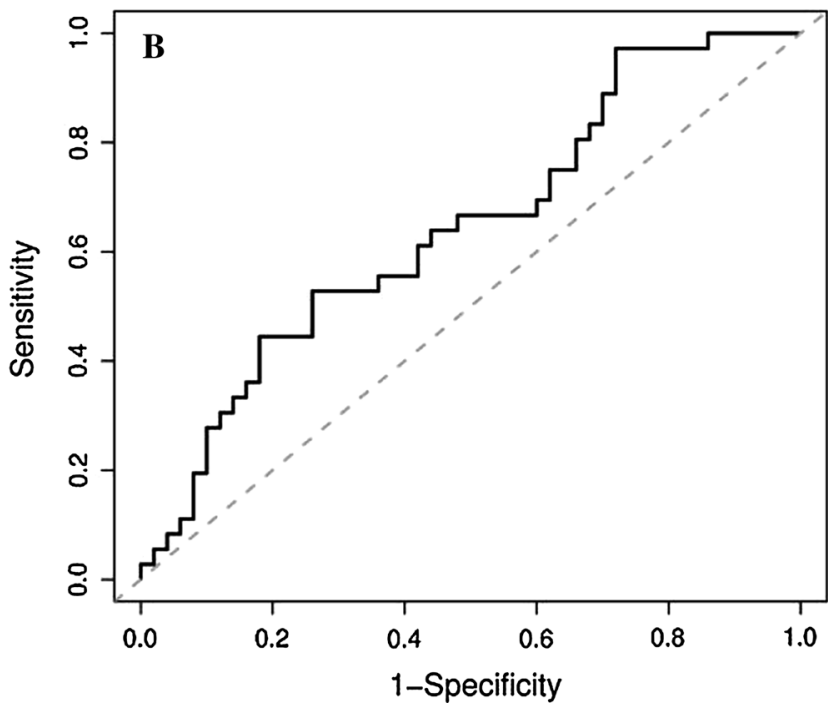

Fig. 3 a ROC curve of patients with colorectal cancer based on RP11-462C24.1 expression in tumor tissues and adjacent normal tissues. b ROC curve of patients with colorectal cancer based on RP11-462C24.1 expression in metastatic tumor tissues and non-metastatic tumor tissues

Table 2 Correlations between RP11-462C24.1 expression and clinical characteristics

\begin{tabular}{|c|c|c|c|c|c|c|}
\hline \multirow[t]{2}{*}{ Characteristics } & \multirow{2}{*}{$\begin{array}{l}\text { Number } \\
\text { of case }\end{array}$} & \multicolumn{4}{|c|}{ RP11-462C24.1 expression } & \multirow[t]{2}{*}{$P$ value } \\
\hline & & $\operatorname{High}(n=32)$ & $\%$ & Low $(n=54)$ & $\%$ & \\
\hline Age (years) & 86 & & & & & 0.132 \\
\hline$<60$ & 43 & 13 & 40.6 & 30 & 55.6 & \\
\hline$\geq 60$ & 43 & 19 & 59.4 & 24 & 44.4 & \\
\hline Gender & & & & & & 0.300 \\
\hline Male & 63 & 25 & 78.1 & 38 & 70.4 & \\
\hline Female & 23 & 7 & 21.9 & 16 & 29.6 & \\
\hline Tumor size $(\mathrm{cm})$ & & & & & & 0.180 \\
\hline$<4$ & 31 & 14 & 43.8 & 17 & 31.5 & \\
\hline$\geq 4$ & 55 & 18 & 56.2 & 37 & 68.5 & \\
\hline Depth of invasion & & & & & & 0.372 \\
\hline $\mathrm{T} 1, \mathrm{~T} 2$ & 16 & 7 & 21.9 & 9 & 16.7 & \\
\hline $\mathrm{T} 3, \mathrm{~T} 4$ & 70 & 25 & 78.1 & 45 & 83.3 & \\
\hline Tumor stage & & & & & & 0.070 \\
\hline I and II & 23 & 12 & 37.5 & 11 & 20.4 & \\
\hline III and IV & 63 & 20 & 62.5 & 43 & 79.6 & \\
\hline Metastasis & & & & & & $0.011 *$ \\
\hline Absent & 36 & 19 & 59.4 & 17 & 31.5 & \\
\hline Present & 50 & 13 & 40.6 & 37 & 68.5 & \\
\hline
\end{tabular}

$* P<0.05$

with CRC by RT-PCR assay. We are particularly interested in one of those six lncRNAs, RP11-462C24.1, because its expression level was decreased with the malignant degree of CRC increased. Based on RT-PCR data of 86 patients, we investigated the association of RP11-462C24.1 expression level with clinicopathological characteristics and prognosis in CRC. To our knowledge, this is the first time to report the dysregulated expression pattern of 
Table 3 Univariate analysis of clinicopathological factors for disease-specific survival

\begin{tabular}{lllll}
\hline Variable & $n$ & Hazard ratio & $95 \%$ CI & $P$ value \\
\hline Age (years) & & & & 0.854 \\
$\quad<60$ & 43 & 1 & & \\
$\geq 60$ & 43 & 1.074 & $0.504-2.284$ & \\
Gender & & & & 0.892 \\
$\quad$ Male & 63 & 1 & & \\
Female & 23 & 0.942 & $0.398-2.23$ & \\
Tumor size (cm) & & & & 0.698 \\
$\quad<4$ & 31 & 1 & & \\
$\quad \geq 4$ & 55 & 0.859 & $0.399-1.852$ & \\
Invasion depth & & & & 0.655 \\
$\quad 1,2$ & 16 & 1 & & \\
3,4 & 70 & 0.813 & $0.328-2.015$ & \\
Distant metastasis & & & & $0.0005^{*}$ \\
Absent & 36 & 1 & & \\
Present & 50 & 8.444 & $2.535-28.13$ & \\
Tumor stage & & & & $0.015^{*}$ \\
I,II & 23 & 1 & & \\
III,IV & 63 & 5.996 & & \\
RP11-462C24.1 & & & & \\
Low & 54 & 1 & & \\
High & 32 & 0.044 & & \\
\hline
\end{tabular}

$* P<0.05$

Table 4 Multivariate analysis of clinicopathological factors for disease-specific survival

\begin{tabular}{|c|c|c|c|}
\hline Variable & $\begin{array}{l}\text { Hazard } \\
\text { ratio }\end{array}$ & $95 \% \mathrm{CI}$ & $P$ value \\
\hline $\begin{array}{l}\text { Distant metastasis (present/ } \\
\text { absent) }\end{array}$ & 4.983 & $1.287-19.292$ & $0.020^{*}$ \\
\hline Tumor stage (III,IV/I,II) & 1.870 & $0.369-9.472$ & 0.449 \\
\hline RP11-462C24.1 (high/low) & 0.056 & $0.007-0.420$ & $0.005^{*}$ \\
\hline
\end{tabular}

$* P<0.05$

RP11-462C24.1 in CRC. More important, we found that RP11-462C24.1 expression level was significantly associated with the state of patients' distant metastasis $(P=0.028)$ and patients' survival. Furthermore, patients with low RP11$462 \mathrm{C} 24.1$ expression level have poor prognosis.

The RP11-462C24.1, a type of IncRNA consisting of four exons with a length of $1,136 \mathrm{bp}$, locates in chr4q25. Ribosomal Protein L34 (RPL34), a leukemia-associated protein, is located head to head with RP11-462C24.1 [32]. It was reported that RPL34 was up-regulated in metastatic breast cancer [33]. RPL34 was also shown highly expressed in the colon cancer line RKO (a human colon cancer line). Thus, RPL34 was reported as a driver gene to promote RKO cell line proliferation [34]. However, RP11-

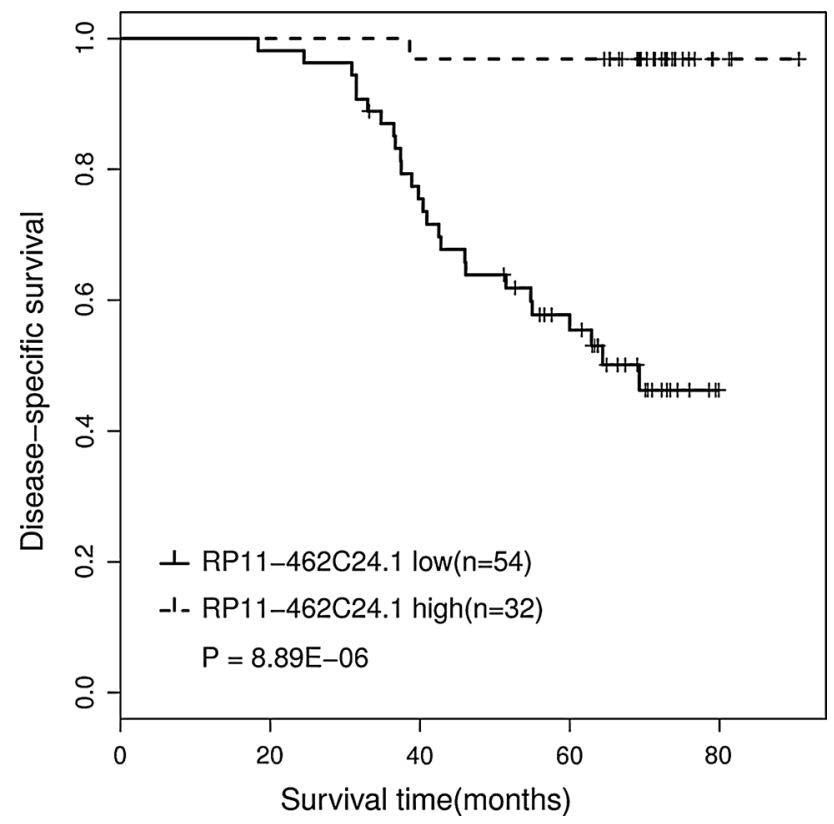

Fig. 4 Kaplan-Meier survival curves of patients with colorectal cancer based on RP11-462C24.1 expression status

462C24.1 was shown to be down-regulated in CRC in this study. Thus, it indicated that RP11-462C24.1, as an antisense transcript of RPL34, may involve in metastasis of CRC by negatively regulating RPL34 expression at the level of chromatin modification, transcription and posttranscriptional processing [10].

LncRNAs may function as oncogenes and tumor suppressors in cancer just like protein-coding genes. A tumor suppressor gene is broadly thought to normally inhibit tumor initiation and progression. And inactivation of tumor suppressor is prone to tumorigenesis. Several studies have demonstrated the potential role of IncRNAs as tumor suppressors. The maternally expressed gene 3 (MEG3) was the first lncRNA proposed to function as a tumor suppressor [35]. The MEG3 gene was detected in many normal human tissues, whereas it was not detected in any nonfunctioning adenomas of gonadotroph origin nor in a range of human cancer cell lines [36-39]. Moreover, ectopic expression of MEG3 was found to suppress the growth of several human cancer cell lines, further supporting the role of MEG3 as a tumor suppressor [36]. Functionally, MEGs has been implicated to interact with p53, a well-known tumor suppressor gene, and inhibit cell proliferation in vitro $[36,40]$. Recently, LOC285194 was reported as a potential tumor suppressor in CRC. Its expression level was significantly lower in CRC and cancer cell line [41]. In present study, we found the expression level of RP11462C24.1, a lncRNA whose function was previously unknown, decreased as the malignant degree of CRC 
increased, which indicated RP11-462C24.1 was a potential tumor suppressor lncRNA.

Though some LncRNAs have reported involving in the progression and development of tumors, the underlying molecular mechanism is still unclearly elucidated. Therefore, in the future, we will investigate the mechanisms of the low expression of RP11-462C24.1 in CRC, and whether and how it functions as a tumor suppressor gene.

In conclusion, we reported a novel dysregulated lncRNA in CRC, RP11-462C24.1, whose expression decreased with the development of CRC. The expression of RP11462C24.1 has a good potential to distinguish CRC tissues from normal tissues, expressed by the large AUC of ROC (0.76). RP11-462C24.1 expression can also distinguish CRC tissues with metastasis from CRC tissues without metastasis, in which AUC of ROC is 0.62. Additionally, we revealed that RP11-462C24.1 expression significantly correlated with distant metastasis. We also demonstrated that RP11-462C24.1 expression was a predictor for patients' survival as implied by Kaplan-Meier analysis. Moreover, univariate analysis revealed that three clinicopathological factors of patients with CRC: metastasis, tumor stage, and RP11-462C24.1 expression were significantly associated with patients' survival. However, only metastasis and RP11-462C24.1 expression were significant in multivariate analysis, which indicated that RP11462C24.1 expression was an independent prognostic indicator for survival of patients with CRC in addition to distant metastasis.

Acknowledgments This work was supported by grants from the Shanghai Municipal Commission of Health and Family Planning (number 20134065). This work was supported in part by the following grants from the National Natural Science Foundation of China (No. 81001055). We thank all of the participants in this study for their contributions. Finally, we thank Peter Wiernik and anonymous reviewer for comments on the manuscript, and Mingbo Yin for critical reading.

Conflict of interest The authors have declared that no conflict of interests exist.

Open Access This article is distributed under the terms of the Creative Commons Attribution License which permits any use, distribution, and reproduction in any medium, provided the original author(s) and the source are credited.

\section{References}

1. Jemal A, Bray F, Center MM, Ferlay J, Ward E, Forman D. Global cancer statistics. CA Cancer J Clin. 2011;61(2):69-90.

2. Zauber AG, Winawer SJ, O'Brien MJ, Lansdorp-Vogelaar I, van Ballegooijen M, Hankey BF, et al. Colonoscopic polypectomy and long-term prevention of colorectal-cancer deaths. N Engl J Med. 2012;366(8):687-96.
3. Edwards BK, Ward E, Kohler BA, Eheman C, Zauber AG, Anderson RN, et al. Annual report to the nation on the status of cancer, 1975-2006, featuring colorectal cancer trends and impact of interventions (risk factors, screening, and treatment) to reduce future rates. Cancer. 2010;116(3):544-73.

4. Cunningham D, Atkin W, Lenz HJ, Lynch HT, Minsky B, Nordlinger B, et al. Colorectal cancer. Lancet. 2010;375(9719): 1030-47.

5. Hayashi N, Ito I, Yanagisawa A, Kato Y, Nakamori S, Imaoka S, et al. Genetic diagnosis of lymph-node metastasis in colorectal cancer. Lancet. 1995;345(8960):1257-9.

6. McMillan DC, McArdle CS. Epidemiology of colorectal liver metastases. Surg Oncol. 2007;16(1):3-5.

7. Park JS, Son KR, Na DG, Kim E, Kim S. Performance of preoperative sonographic staging of papillary thyroid carcinoma based on the sixth edition of the AJCC/UICC TNM classification system. AJR Am J Roentgenol. 2009;192(1):66-72.

8. Ponting CP, Oliver PL, Reik W. Evolution and functions of long noncoding RNAs. Cell. 2009;136(4):629-41.

9. Maruyama R, Suzuki H. Long noncoding RNA involvement in cancer. Biochem Mol Biol Rep. 2012;45(11):604-11.

10. Mercer TR, Dinger ME, Mattick JS. Long non-coding RNAs: insights into functions. Nat Rev Genet. 2009;10(3):155-9.

11. Wapinski O, Chang HY. Long noncoding RNAs and human disease. Trends Cell Biol. 2011;21(6):354-61.

12. Wu SC, Kallin EM, Zhang Y. Role of H3K27 methylation in the regulation of lncRNA expression. Cell Res. 2010;20(10): 1109-16.

13. Esteller M. Non-coding RNAs in human disease. Nat Rev Genet. 2011;12(12):861-74.

14. Mattick JS. The genetic signatures of noncoding RNAs. PLoS Genet. 2009;5(4):e1000459.

15. Kogo R, Shimamura T, Mimori K, Kawahara K, Imoto S, Sudo T, et al. Long noncoding RNA HOTAIR regulates polycombdependent chromatin modification and is associated with poor prognosis in colorectal cancers. Cancer Res. 2011;71(20):6320-6.

16. Gupta RA, Shah N, Wang KC, Kim J, Horlings HM, Wong DJ, et al. Long non-coding RNA HOTAIR reprograms chromatin state to promote cancer metastasis. Nature. 2010;464(7291): 1071-6.

17. Tsai MC, Manor O, Wan Y, Mosammaparast N, Wang JK, Lan F, et al. Long noncoding RNA as modular scaffold of histone modification complexes. Science. 2010;329(5992):689-93.

18. Yang Z, Zhou L, Wu LM, Lai MC, Xie HY, Zhang F, et al. Overexpression of long noncoding RNA HOTAIR predicts tumor recurrence in hepatocellular carcinoma patients following liver transplantation. Ann Surg Oncol. 2011;18(5):1243-50.

19. Kim K, Jutooru I, Chadalapaka G, Johnson G, Frank J, Burghardt $\mathrm{R}$, et al. HOTAIR is a negative prognostic factor and exhibits prooncogenic activity in pancreatic cancer. Oncogene. 2013;32(13): $1616-25$

20. Ji P, Diederichs S, Wang W, Böing S, Metzger R, Schneider PM, et al. MALAT-1, a novel noncoding RNA, and thymosin beta4 predict metastasis and survival in early-stage non-small cell lung cancer. Oncogene. 2003;22(39):8031-41.

21. Lin R, Maeda S, Liu C, Karin M, Edgington TS. A large noncoding RNA is a marker for murine hepatocellular carcinomas and a spectrum of human carcinomas. Oncogene. 2007;26(6): $851-8$.

22. Tripathi V, Ellis JD, Shen Z, Song DY, Pan Q, Watt AT, et al. The nuclear-retained noncoding RNA MALAT1 regulates alternative splicing by modulating SR splicing factor phosphorylation. Mol Cell. 2010;39(6):925-38.

23. Yang L, Lin C, Liu W, Zhang J, Ohgi KA, Grinstein JD, et al. NcRNA- and Pc2 methylation-dependent gene relocation 
between nuclear structures mediates gene activation programs. Cell. 2011;147(4):773-88.

24. Graham LD, Pedersen SK, Brown GS, Ho T, Kassir Z, Moynihan AT, et al. Colorectal neoplasia differentially expressed (CRNDE), a novel gene with elevated expression in colorectal adenomas and adenocarcinomas. Genes Cancer. 2011;2(8): $829-40$.

25. Ellis BC, Molloy PL, Graham LD. CRNDE: a long non-coding RNA involved in Cancer, neurobiology, and development. Front Genet. 2012;3:270

26. Zhang X, Sun S, Pu JK, Tsang AC, Lee D, Man VO, et al. Long non-coding RNA expression profiles predict clinical phenotypes in glioma. Neurobiol Dis. 2012;48(1):1-8.

27. Ellis BC, Graham LD, Molloy PL. CRNDE: a long non-coding RNA responsive to insulin/IGF signaling, regulates genes involved in central metabolism. Biochim Biophys Acta. 2014; 1843(2):372-86.

28. Xu C, Yang M, Tian J, Wang X, Li Z. MALAT-1: a long noncoding RNA and its important $3^{\prime}$ end functional motif in colorectal cancer metastasis. Int J Oncol. 2011;39(1):169-75.

29. Gutschner T, Hämmerle M, Diederichs S. MALAT1-a paradigm for long noncoding RNA function in cancer. $\mathrm{J}$ Mol Med (Berl). 2013;91(7):791-801.

30. Nagaraj SH, Reverter A. A Boolean-based systems biology approach to predict novel genes associated with cancer: application to colorectal cancer. BMC Syst Biol. 2011;5:35.

31. Poliseno L, Salmena L, Zhang J, Carver B, Haveman WJ, Pandolfi PP. A coding-independent function of gene and pseudogene mRNAs regulates tumour biology. Nature. 2010;465(7301): 1033-8.

32. Ota T, Suzuki Y, Nishikawa T, Otsuki T, Sugiyama T, Irie R, et al. Complete sequencing and characterization of 21,243 fulllength human cDNAs. Nat Genet. 2004;36(1):40-5.
33. Zucchi I, Mento E, Kuznetsov VA, Scotti M, Valsecchi V, Simionati B, et al. Gene expression profiles of epithelial cells microscopically isolated from a breast-invasive ductal carcinoma and a nodal metastasis. Proc Natl Acad Sci USA. 2004;101(52): 18147-52.

34. Kim HS, Kuwano Y, Zhan M, Pullmann R Jr, Mazan-Mamczarz $\mathrm{K}, \mathrm{Li} \mathrm{H}$, et al. Elucidation of a C-rich signature motif in target mRNAs of RNA-binding protein TIAR. Mol Cell Biol. 2007; 27(19):6806-17.

35. Gibb EA, Brown CJ, Lam WL. The functional role of long noncoding RNA in human carcinomas. Mol Cancer. 2011;10:38.

36. Zhang X, Zhou Y, Mehta KR, Danila DC, Scolavino S, Johnson $\mathrm{SR}$, et al. A pituitary-derived MEG3 isoform functions as a growth suppressor in tumor cells. J Clin Endocrinol Metab. 2003;88(11):5119-26.

37. Astuti D, Latif F, Wagner K, Gentle D, Cooper WN, Catchpoole D, et al. Epigenetic alteration at the DLK1-GTL2 imprinted domain in human neoplasia: analysis of neuroblastoma, phaeochromocytoma and Wilms' tumour. Br J Cancer. 2005;92(8): 1574-80.

38. Braconi C, Kogure T, Valeri N, Huang N, Nuovo G, Costinean S, et al. MicroRNA-29 can regulate expression of the long noncoding RNA gene MEG3 in hepatocellular cancer. Oncogene. 2011;30(47):4750-6.

39. Wang P, Ren Z, Sun P. Overexpression of the long non-coding RNA MEG3 impairs in vitro glioma cell proliferation. J Cell Biochem. 2012;113(6):1868-74.

40. Zhou Y, Zhong Y, Wang Y, Zhang X, Batista DL, Gejman R, et al. Activation of p53 by MEG3 non-coding RNA. J Biol Chem. 2007;282(34):24731-42.

41. Qi P, Xu MD, Ni SJ, Huang D, Wei P, Tan C, et al. Low expression of LOC285194 is associated with poor prognosis in colorectal cancer. J Transl Med. 2013;11(1):122. 